\title{
Comparison of two regimens of vitamin D supplementation for vitamin D-deficient neonates
}

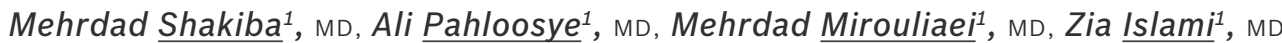

\begin{abstract}
INTRODUCTION Vitamin D deficiency is common in pregnant women, and supplementation of vitamin D is necessary for the infants of these women. This study explored the efficacy of an alternative way of vitamin D supplementation in an area with a high prevalence of vitamin D deficiency in mothers.

METHODS This was a non-randomised clinical trial conducted in 2010 in Yazd, Iran. Full-term healthy infants born to vitamin $D$-deficient mothers $(n=82)$ were divided into the high-dose regimen (HDR; single oral bolus $30,000 \mathrm{IU}$ vitamin $D_{3}, n=34$ ) and the standard-dose regimen (SDR; $400 \mathrm{IU} /$ day vitamin $D_{3}$ within two weeks of life, $n=48$ ) groups. 25 -hydroxyvitamin D (25OHD) was measured using chemiluminescent immunoassays, and $25 \mathrm{OHD}$ level $>20 \mathrm{ng} / \mathrm{mL}$ was deemed sufficient.

RESULTS Over $90 \%$ of infants in the HDR group attained vitamin D sufficiency within one month, while comparable sufficiency was reached in the SDR group only after four months. At two months, the proportion of infants attaining $25 \mathrm{OHD}>30 \mathrm{ng} / \mathrm{mL}$ was $93.3 \%$ and $27.9 \%$ in the HDR and SDR groups, respectively $(p=0.003)$. None of our infants achieved $250 \mathrm{HD}$ levels $>100 \mathrm{ng} / \mathrm{mL}$.

CONCLUSION For infants born to vitamin D-deficient mothers, oral supplementation of 30,000 IU vitamin $D_{3}$ during the first month of life, followed by a routine recommended dose of $400 \mathrm{IU} / \mathrm{day}$, should be considered. The four-month lag for attaining vitamin D sufficiency in $90 \%$ of infants in the SDR group may have clinical implications and should be further investigated.
\end{abstract}

Keywords: infant, supplement, vitamin D

\section{INTRODUCTION}

Breast milk is the single best food source for infants. However, the breast milk of mothers on normal diets only contains 25-78 IU/L of vitamin D and is therefore insufficient as a source of vitamin D for infants. ${ }^{(1)}$ The optimal blood level of 25-hydroxyvitamin D (25OHD) in newborns is as yet unknown. ${ }^{(1,2)}$ However, it is generally accepted that a serum $25 \mathrm{OHD}$ level of $11 \mathrm{ng} / \mathrm{mL}$ in newborns is sufficient to prevent rickets $^{(2,3)}$ and that a serum $25 \mathrm{OHD}$ level of more than $30 \mathrm{ng} / \mathrm{mL}$ is necessary to control secondary hyperparathyroidism as well as increase calcium absorption. Serum 25OHD levels of more than $30 \mathrm{ng} / \mathrm{mL}$ are accepted as ideal, and 25OHD levels less than $20 \mathrm{ng} / \mathrm{mL}$ are considered deficient. ${ }^{(3)}$

To provide adequate levels of vitamin D to newborns, the American Academy of Pediatrics (AAP) recommends universal oral vitamin D supplementation at $400 \mathrm{IU} /$ day for all breastfeeding infants. (1) This dosage, believed by many experts as the minimal appropriate dose for infants, ${ }^{(2,3)}$ is generally thought to be sufficient to prevent rickets and vitamin D deficiency in neonates, although in certain situations, higher vitamin D supplementation may be necessary. According to some studies, 25OHD concentrations in umbilical cord blood at the time of delivery ranges between $68 \%$ and $108 \%$ of maternal $25 \mathrm{OHD}$ levels, and therefore infants born to vitamin D-deficient mothers would have low reserves of vitamin $D^{(4,5)}$ In the present study, we aimed to determine whether the recommended dose of $400 \mathrm{IU} /$ day of vitamin D for newborns was optimal for infants born to vitamin D-deficient mothers in Iran.

Vitamin D insufficiency is common among healthy pregnant women worldwide, especially in the Middle East. ${ }^{(5)}$ For this reason, we hypothesised that the current recommendation of 400 IU/day of vitamin D supplementation for neonates and infants may not be adequate for those born to vitamin Ddeficient mothers. With vitamin D supplements being administered at the currently recommended doses, it is possible that a large proportion of vitamin D-deficient newborns may remain under-supplemented or deficient up to a period of at least 1-2 months after birth. Although the period of such vitamin $\mathrm{D}$ deficiency may be short, any delay in reaching sufficient levels of vitamin D during the crucial perinatal period, when infants are exposed to various antigens, may have significant implications. Some studies indicate that individuals with low vitamin D levels may be more prone to unbalanced immune responses or diseases in the future. ${ }^{(6-10)}$

In view of such studies, we attempted to determine the time needed to attain sufficient levels of serum 25OHD in vitamin D-deficient newborns on two different vitamin D

Department of Pediatrics, Shahid Sadoughi University of Medical Science, Yazd, Iran Correspondence: Dr Mehrdad Shakiba, Assistant Professor, Department of Pediatrics, Shahid Sadoughi University of Medical Science, Avicenna Street, Yazd, Iran.shakiba@ssu.ac.ir 


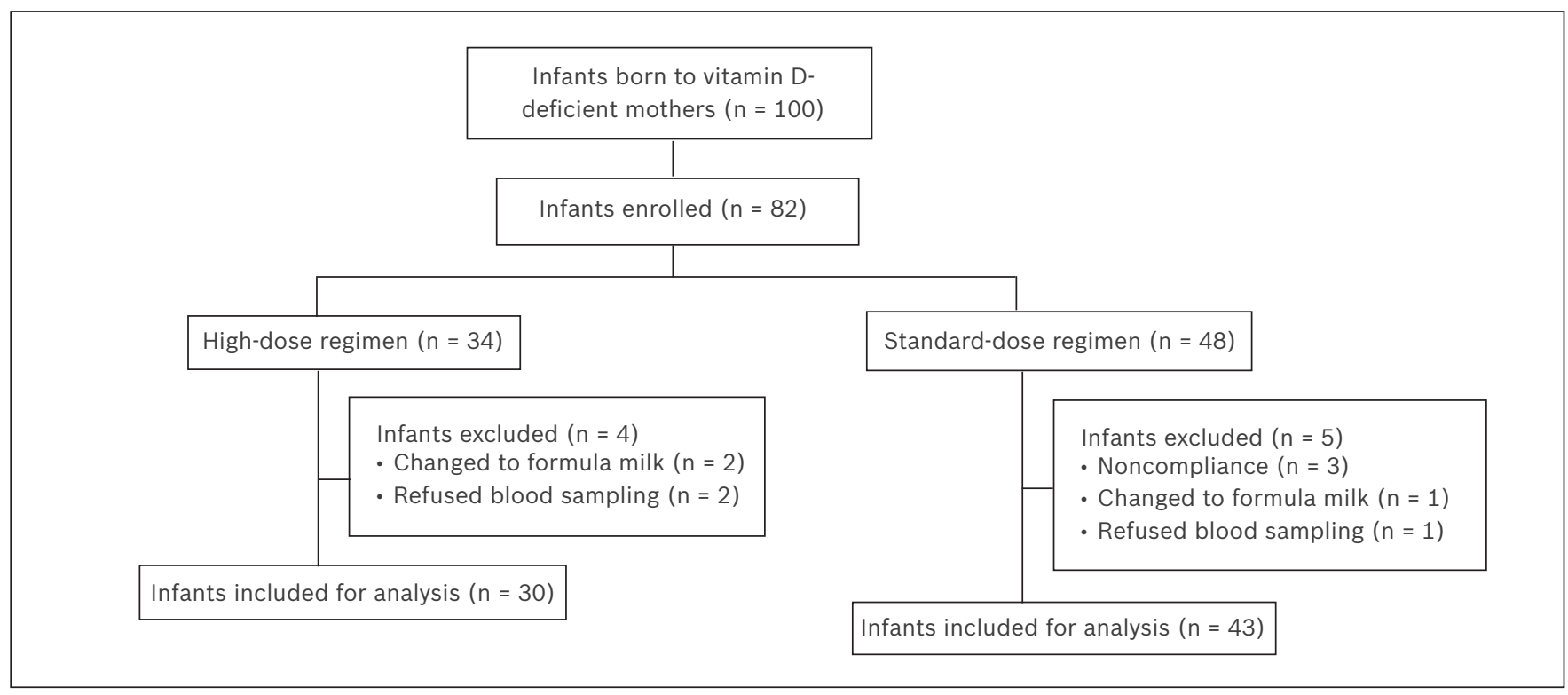

Fig. 1 Chart shows the distribution of infants in the study.

supplementation regimens - infants receiving the recommended 400 IU/day within two weeks of life were categorised as being on standard-dose regimen (SDR), while those who received $30,000 \mathrm{IU} / \mathrm{month}$ as a single oral bolus were grouped as infants on high-dose regimen (HDR).

\section{METHODS}

In a quasi-experimental clinical trial without randomisation, 82 healthy, breast-fed, full-term neonates born to vitamin Ddeficient Iranian mothers were selected and enrolled in the study in 2010. The participants were all recruited from primary care clinics or general maternity hospitals in Yazd, Iran. All the pregnancies were uneventful and the mothers did not receive any high-dose supplements of vitamin D (no more than $400 \mathrm{IU}$ of vitamin $D_{3}$ ) during the gestation and nursing periods. Informed consent was obtained from all the neonates' parents, who were instructed regarding the nature of study. Approval was also obtained from the ethics committee at the Yazd University of Medical Science, Yazd, Iran.

The neonates were allocated into either one of two groups - the test group (HDR) or the control group (SDR) - and serum 25OHD levels were assessed to determine when sufficiency of vitamin D was reached. In the first week of life, 34 infants in the HDR group (with serum 25OHD level $<20 \mathrm{ng} / \mathrm{mL}$ ) received a single-dose 30,000 IU of oral vitamin $\mathrm{D}_{3}$ (Alhavy Drug Company, Tehran, Iran) The serum 25OHD levels in these infants were determined at the end of each month in order to ascertain whether they had attained a $25 \mathrm{OHD}$ level $>30 \mathrm{ng} / \mathrm{mL}$. Infants who attained vitamin D sufficiency were thereafter supplemented using standard doses of 400 IU/day vitamin $D_{3}$ drops. Infants who did not attain vitamin D sufficiency after one month of HDR supplementation received an additional single dose of 30,000 IU oral vitamin $\mathrm{D}_{3}$ and were reassessed one month later. Infants in the control SDR group $(n=48)$ received the recommended $400 \mathrm{IU} /$ day of vitamin $\mathrm{D}_{3}$ within
15 days of life. The serum 25OHD levels in these infants were assessed twice (at the age of two and four months) and the results were compared to determine whether vitamin D sufficiency was attained by two and four months. These findings were then compared with those from the HDR group.

Infants who consumed less than $10 \%$ of the recommended vitamin D dosage (i.e. three days in a month), changed to formula feeding (over $200 \mathrm{~mL} /$ day) or refused blood sampling were not included in the final analysis. Fig. 1 shows the distribution of infants in the study.

Blood samples were obtained from each infant and 25OHD levels were determined using a chemiluminescent immunoassay (LIAISON XL ${ }^{\circledR}$; DiaSorin, Saluggia, Italy). Samples were quickly centrifuged and refrigerated in order to not confound the study results. Aiming to maintain the accuracy of the laboratory checking system, $20 \%$ of all serum vitamin D results were double-checked by laboratory experts and some samples were randomly measured by another reference laboratory during the entire study. The intra- and interassay coefficients of variance were found to be $11 \%$ and $13 \%$, respectively.

\section{RESULTS}

In total, 41 boys and 32 girls were included in our study, with birth weights ranging between 2,600 g and 4,200 g. There was no difference between the mean birth weights of infants among the HDR and SDR groups ( $p=0.67$ ). Of the 34 infants in the HDR group, 4 were excluded from the analysis. Among the remaining 30 infants, 28 (93\%) attained $25 \mathrm{OHD}$ level $>20 \mathrm{ng} / \mathrm{mL}$ within one month, while $2(7 \%)$ infants reached this level within two months of vitamin D supplementation (Fig. 2). In other words, within two months of vitamin D supplementation in the HDR group, all the infants reached serum 25OHD level $>20 \mathrm{ng} / \mathrm{mL}$ and 28 (93.3\%) infants attained ideal 25OHD level of $>30 \mathrm{ng} / \mathrm{mL}$ (Fig. 3 \& Table I). A majority of infants (66\%) attained ideal 25OHD levels within one month of HDR 
Table I. Infants in the high-dose regimen (HDR) and standarddose regimen (SDR) groups with vitamin D sufficiency and insufficiency after two months of supplementation.

\begin{tabular}{lcc}
\hline Group & \multicolumn{2}{c}{ No. (\%) } \\
\cline { 2 - 3 } & $\begin{array}{c}\text { Vitamin D-sufficient } \\
(\mathbf{2 5 O H D}>\mathbf{3 0} \mathbf{n g} / \mathbf{m L})\end{array}$ & $\begin{array}{c}\text { Vitamin D-insufficient } \\
\mathbf{( 2 5 O H D}<\mathbf{3 0} \mathbf{n g} / \mathbf{m L})\end{array}$ \\
\hline HDR $(n=30)$ & $28(93.3)$ & $2(6.7)$ \\
SDR $(n=43)$ & $12(27.9)$ & $31(72.1)$ \\
\hline
\end{tabular}

$p$-value was statistically significant $(p=0.003$; chi-square test).

25OHD: 25-hydroxyvitamin D

supplementation and all infants achieved an ideal 25OHD level by four months (Fig. 3).

Of the 48 infants in the SDR group, 5 were excluded from the analysis. Among the remaining 43 infants, 28 attained serum $25 \mathrm{OHD}$ level $>20 \mathrm{ng} / \mathrm{mL}$ within two months of standard-dose vitamin $\mathrm{D}_{3}$ supplementation, while 2 infants reached ideal levels within four months of supplementation. Fig. 2 shows the proportion of infants who were not vitamin D-deficient in terms of serum $25 \mathrm{OHD}$ level (> $20 \mathrm{ng} / \mathrm{mL}$ ) in the HDR and SDR groups during the four months of study. Fig. 3 provides the proportion of infants who reached the ideal serum 25OHD level (> $30 \mathrm{ng} / \mathrm{mL}$ ) in the HDR and SDR groups during this period. Table I compares the number of infants in the HDR and SDR groups with vitamin D sufficiency and insufficiency two months after supplementation had begun $(p=0.003$; chi-square test).

Fig. 4, which provides the mean serum 25OHD levels of infants in the SDR $(32 \pm 3.5 \mathrm{ng} / \mathrm{mL})$ and HDR $(46 \pm 4.9 \mathrm{ng} / \mathrm{mL})$ groups when they were not deficient, shows the statistically significant difference between the levels of serum 25OHD in the two groups ( $p=0.004$; $t$-test).

\section{DISCUSSION}

We found that high-dose supplementation of vitamin $D_{3}$ at birth was both a safe and effective means of supplying adequate amounts of the vitamin to neonates born to vitamin D-deficient mothers, and that these infants achieved vitamin D sufficiency sooner than infants receiving standard-dose vitamin $\mathrm{D}_{3}$ supplementation. In the HDR group, $90 \%$ of infants achieved 25OHD level $>20 \mathrm{ng} / \mathrm{mL}$ within one month with just a single dose administered. In comparison, the percentage of infants in the SDR group who had achieved similar levels was lower than that in the HDR group, even after the second month of supplementation. A high prevalence of low vitamin D levels in neonates during the perinatal period has previously been reported. ${ }^{(11)}$ A few studies have indicated that vitamin D -deficient infants attain an acceptable level of serum 25OHD in the first month of life upon administration of the currently recommended doses for vitamin D supplementation. ${ }^{(12,13)}$ Some others have even recommended alternative doses in the range of 800-1,000 IU/day following birth, in order to attain acceptable levels of serum 25OHD..$^{(2,3,12,13)}$ In our study, after four months of supplementation, the percentage of infants who were not deficient (i.e $25 \mathrm{OHD}$ level $>20 \mathrm{ng} / \mathrm{mL}$ ) in

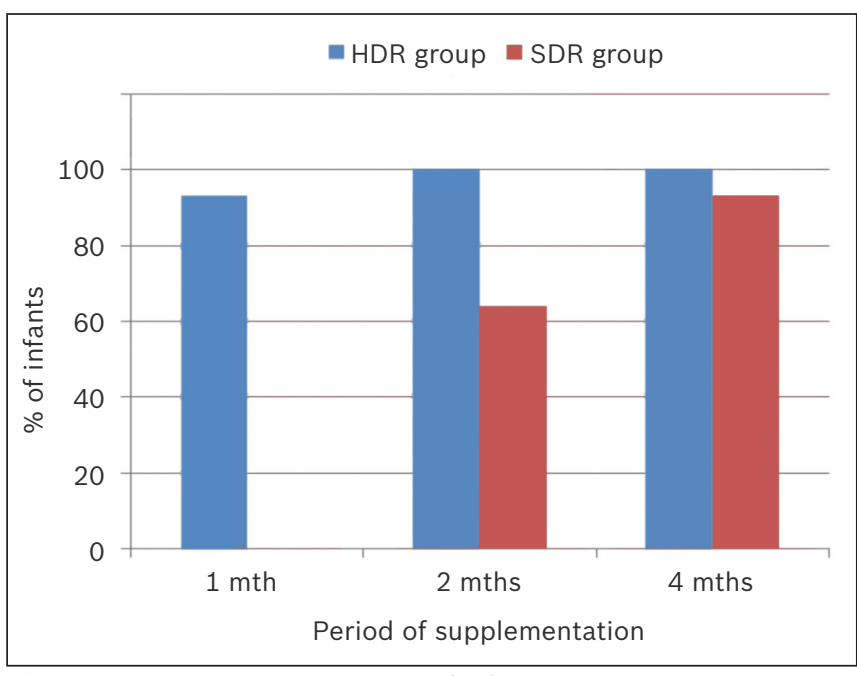

Fig. 2 Graph shows the proportion of infants in the high-dose regimen (HDR) and standard-dose regimen (SDR) groups who attained serum 25 -hydroxyvitamin $D$ levels $>20 \mathrm{ng} / \mathrm{mL}$ after receiving vitamin $D$ supplementation for varying periods of time.

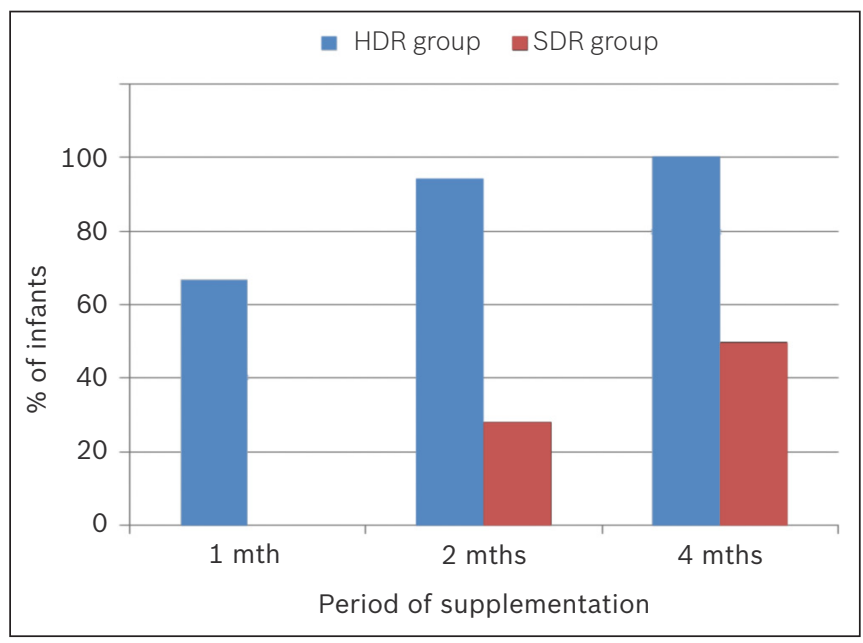

Fig. 3 Graph shows the proportion of infants in the high-dose regimen (HDR) and standard-dose regimen (SDR) groups who attained the ideal serum level of 25 -hydroxyvitamin $D$ of $>30 \mathrm{ng} / \mathrm{mL}$ over varying periods of time.

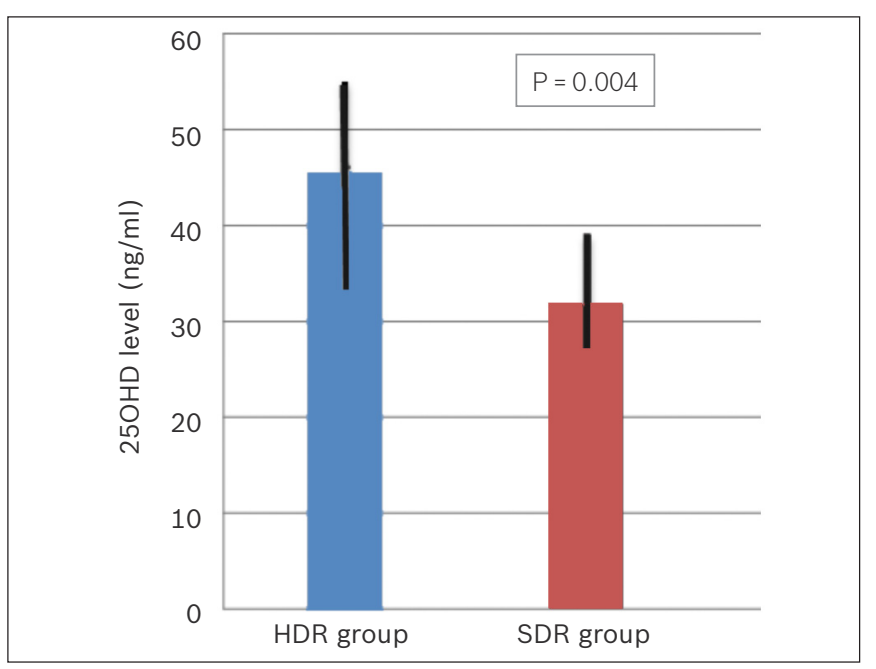

Fig. 4 Mean serum 25-hydroxyvitamin D levels of infants in the high-dose regimen (HDR) and standard-dose regimen (SDR) groups who attained vitamin D sufficiency. 
the HDR and SDR groups was comparable. This was similar to the findings of Wagner et al, whose study revealed that $70 \%$ of infants were vitamin D-deficient at birth; however, in this study, vitamin D supplementation was started slightly late (after one month of life). ${ }^{(14)}$ However, according to Abrams et al, 25OHD levels in deficient infants (25OHD levels $<20 \mathrm{ng} / \mathrm{mL}$ ) receiving $400 \mathrm{IU} /$ day of vitamin $\mathrm{D}$ rose to $>20 \mathrm{ng} / \mathrm{mL}$ within three months of life, and half of their cohort had 25OHD levels $<30 \mathrm{ng} / \mathrm{mL}$. ${ }^{(15)}$ A Turkish study by Onal et al also found that $30 \%$ of infants receiving vitamin D supplementation of $400 \mathrm{IU} /$ day remained vitamin D-deficient between 2 and 6 months of life. ${ }^{(16)}$

Such variations in the literature may be accounted for, to a certain degree, by differences between the various study populations or the role played by other sources of vitamin D, such as sun exposure. ${ }^{(17)}$ While studies have reported a time lag between birth and the achievement of vitamin D sufficiency by vitamin D-deficient newborns, the significance of this period of insufficiency has not been established. For instance, Kim et al suggested that the period of insufficiency would not necessarily lead to acute skeletal problems such as rickets. ${ }^{(18)}$ Greer, meanwhile, highlighted the need to establish the nonskeletal clinical implications of this period. ${ }^{(19)}$

While research on the implications of vitamin D deficiency in the immediate perinatal period is ongoing, it would be sensible to institute interventions that could narrow the insufficiency period. The best way of achieving this would be to supply adequate vitamin D to mothers during pregnancy and the lactation period. However, in areas where maternal vitamin D deficiency is highly prevalent and there is limited access to such mothers prior to delivery, HDR should be considered as a quick and efficient means of attaining sufficient levels of vitamin D in infants. ${ }^{(20,21)}$ In infants, the safe upper limit for serum $25 \mathrm{OHD}$ is accepted as serum level $<100 \mathrm{ng} / \mathrm{mL}$. ${ }^{(3)}$ Although higher doses of vitamin $\mathrm{D}$ are well tolerated by infants, further studies will be needed to substantiate the relationship between such dosage and its side effects, if any. ${ }^{(22)}$

The present study had several limitations. We were unable to monitor sun exposure in our infants and mothers. As it is customary in Iran to prevent the exposure of infants to the sun in the early months of life, we assumed that sun exposure had limited effects in our cohort, especially during the first two months. Furthermore, mothers included in our study had prior limited sun exposure and were all vitamin D deficient, and it is unlikely that they would have changed their lifestyles during the period of study. Vitamin D levels in infants in the SDR group were not determined after the first month of life for two reasons: (a) difficulty of repeatedly obtaining blood from healthy infants; and (b) study conclusions were unlikely to have varied, as the proportion of infants in the SDR group who could be expected to be vitamin $\mathrm{D}$ deficient after the first month of life would only have been higher than numbers seen after two months of such supplementation. The prevalence of maternal vitamin D deficiency is known to be high in the Middle East and therefore our cohort may not be representative of infants worldwide. For this reason, the extrapolation of our results to infants from other countries where vitamin D deficiency is not as prevalent might not be appropriate.

Infants receiving standard vitamin D supplementation must consume the doses daily. As such, healthcare workers do not supervise this dosage and the likelihood of noncompliance with the supplementation regimen is quite high. Our results indicate that, for regions where high maternal vitamin D deficiencies are compounded by limited access to such mothers prior to delivery, vitamin D supplementation via HDR would be a quick and efficient means of attaining vitamin $D$ sufficiency in infants. For infants born to vitamin D-deficient mothers, oral supplementation of 30,000 IU of vitamin $\mathrm{D}_{3}$ as a single dose during the first month of life followed by routine recommended dosage of $400 \mathrm{IU} /$ day would ensure that vitamin D sufficiency is achieved sooner rather than later. In our study, the four-month lag for attaining vitamin D sufficiency noticed in infants from the SDR group might have clinical implications and warrants further investigation. Future studies with larger sample sizes should look to establish the efficacy and consequences of such supplementation.

\section{ACKNOWLEDGEMENTS}

The authors wish to thank all the parents who participated in the study. We are also grateful to Fariba Shamsi, Statistics Division, Faculty of Health, Shahid Sadoughi University of Medical Science, Iran, for her contribution to the statistical analysis of the data. This article was based on the research conducted by $\operatorname{Dr}$ A Pahloosye as part of her dissertation for obtaining a specialist certification in paediatrics, under the supervision of Dr M Shakiba.

\section{REFERENCES}

1. Wagner $\mathrm{CL}$, Greer FR. American Academy of Pediatrics Section on Breastfeeding; American Academy of Pediatrics Committee on Nutrition. Prevention of rickets and vitamin D deficiency in infants, children, and adolescents. Pediatrics 2008; 122:1142-52.

2. No authors listed. Vitamin D supplementation: Recommendations for Canadian mothers and infants. Paediatr Child Health 2007; 12:583-98.

3. Holick MF, Binkley NC, Bischoff-Ferrari HA, et al. Evaluation, treatment, and prevention of vitamin D deficiency: an Endocrine Society clinical practice guideline. J Clin Endocrinol Metab 2011; 96:1911-30.

4. Bowyer L, Catling-Paull C, Diamond T, et al. VitaminD, PTH and calcium levels in pregnant women and their neonates. Clin Endocrinol (Oxf) 2009; 70:372-7.

5. Palacios C, Gonzalez L. Is vitamin D deficiency a major global public health problem? J Steroid Biochem Mol Biol 2013 Nov 12.

6. Novakovic B, Galati JC, Chen A, et al. Maternal vitamin D predominates over genetic factors in determining neonatal circulating vitamin D concentrations. Am J Clin Nutr 2012; 96:188-95.

7. Searing DA, Leung DY. Vitamin D in atopic dermatitis, asthma and allergic diseases. Immunol Allergy Clin North Am 2010; 30:397-409.

8. Kamen DL, Tangpricha V. Vitamin D and molecular actions on the immune system modulation of innate and autoimmunity. J Mol Med (Berl) 2010; 88:441-50. 
9. Bikle DD. Vitamin D regulation of immune function. Vitam Horm 2011; 86:1-21.

10. Hyppönen $E$, Läärä $E$, Reunanen $A$, et al. Intake of vitamin $D$ and risk of type 1 diabetes: a birth-cohort study. Lancet 2001; 358:1500-3.

11. Dijkstra SH, van Beek A, Janssen JW, et al. Prevalence of vitamin D deficiency in newborn infants of high-risk mothers. Arch Dis Child 2007; 92:750-3.

12. Zeghoud F, Vervel C, Guillozo H, et al. Subclinical vitamin D deficiency in neonates: definition and response to vitamin D supplements. Am J Clin Nutr 1997; 65:771-8

13. Vervel C, Zeghoud F, Boutignon $\mathrm{H}$, et al. [Fortified milk and supplements of oral vitamin D. Comparison of the effect of two doses of vitamin D (500 and 1,000 UI/d) during the first trimester of life]. Arch Pediatr 1997; 4:126-32. French.

14. Wagner CL, Howard C, Hulsey TC, et al. Circulating 25-hydroxyvitamin d levels in fully breastfed infants on oral vitamin D supplementation. Int J Endocrinol 2010; 2010:235035.

15. Abrams SA, Hawthorne KM, Rogers SP, Hicks PD, Carpenter TO. Effects of ethnicity and vitamin D supplementation on vitamin D status and changes in bone mineral content in infants. BMC Pediatr 2012; 12:6.
16. Onal H, Adal E, Alpaslan S, Ersen A, Aydin A: Is daily 400 IU of vitamin D supplementation appropriate for every country: a cross-sectional study. Eur J Nutr 2010; 49:395-400.

17. Merewood A, Mehta SD, Grossman X, et al. Vitamin D status among 4-month-old infants in New England: a prospective cohort study. J Hum Lact 2012; 28:159-66.

18. Kim MJ, Na B, No SJ, et al. Nutritional status of vitamin D and the effect of vitamin D supplementation in Korean breast-fed infants. J Korean Med Sci 2010; 25:83-9.

19. Greer FR. 25-Hydroxyvitamin D: functional outcomes in infants and young children. Am J Clin Nutr 2008; 88:529S-533S.

20. Hollis BW, Wagner CL. Vitamin D requirements during lactation: highdose maternal supplementation as therapy to prevent hypovitaminosis $\mathrm{D}$ for both the mother and the nursing infant. Am J Clin Nutr 2004; 80:1752S-8S

21. Haggerty LL. Maternal supplementation for prevention and treatment of vitamin D deficiency in exclusively breastfed infants. Breastfeed Med 2011; 6:137-44.

22. Shakiba M, Sadr S, Nefei Z, et al. Combination of bolus dose vitamin D with routine vaccination in infants: a randomised trial. Singapore Med 2010; 51:440-5.

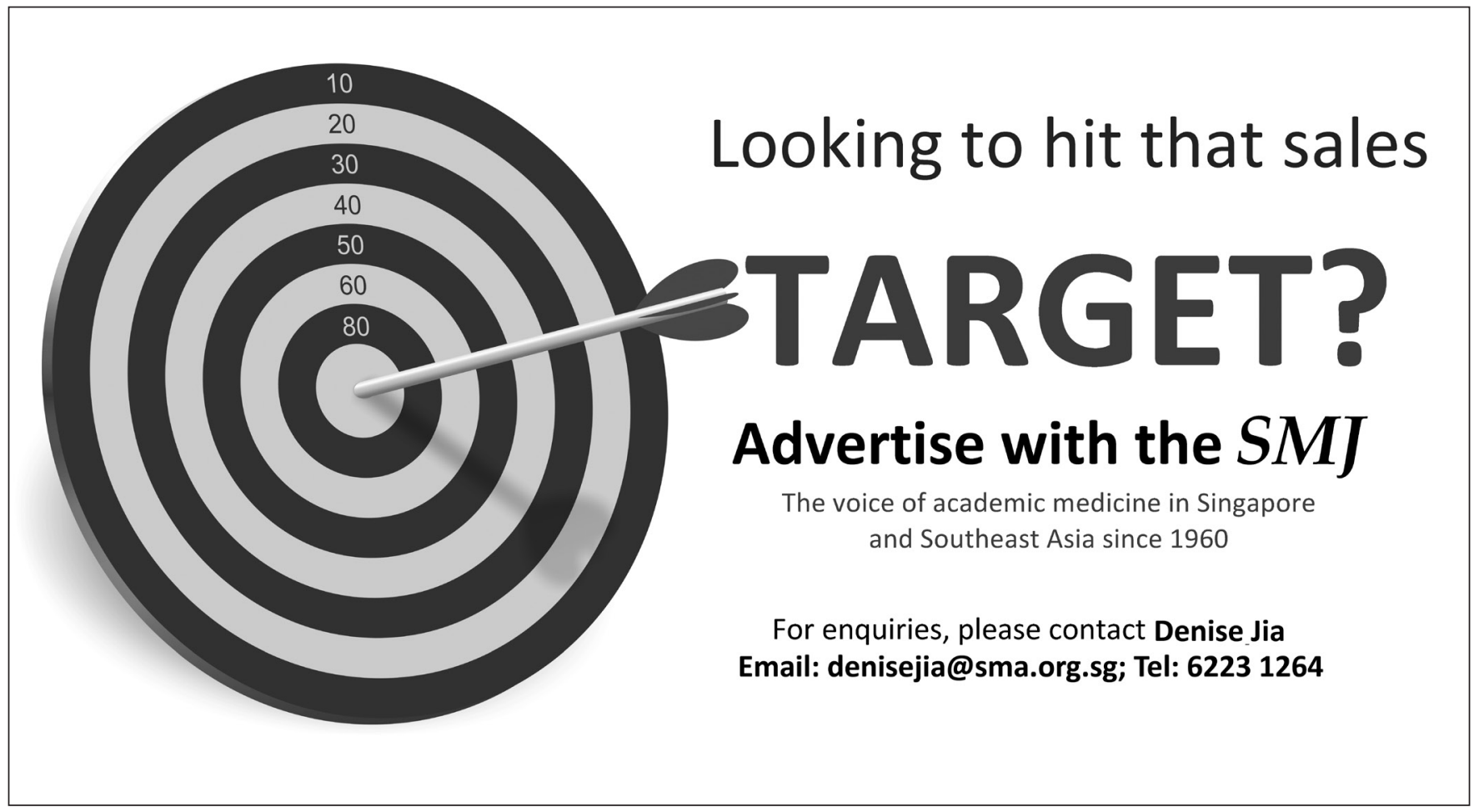

